nem sog. „Brückenfaden“ entlang läuft und dabei immer wieder neue Fäden auf die bereits vorhandenen auflegt und sie in gewissen Abständen daran befestigt. Es mag sein, daß man bei der Analyse der submikroskopischen Struktur fibrilläre Untereinheiten an Spinnfäden entdecken wird, obwohl diese un- ter dem gewöhnlichen Lichtmikroskop homogen erscheinen. Aber es ist sicher falsch, so etwas anzunehmen wie der alte Ré a u m u r, der z. B. meinte, die Kokonfäden setzten sich aus 18 Einzelfäden zusammen, weil sie achtzehnmal so dick seien wie Netzfäden.

\title{
Vergleichende chemische Untersuchungen über die Fibroine von Bombyx mori und Nephila madagascariensis
}

\author{
Von Gerhard Braunitzer und Dieter Wolff \\ Aus dem Max-Planck-Institut für Virusforschung (Biochemische Abteilung) \\ und dem Zoophysiologischen Institut der Universität, Tübingen \\ (Z. Naturforschg. 10 b, 404-408 [1955]; eingegangen am 4. Juli 1955)
}

\begin{abstract}
Die Aminosäure-Zusammensetzung der Fibroine von Nephila madagascariensis und Bombyx mori wurde vergleichend untersucht. Weiterhin wurden bei beiden Fibroinen die Endgruppen nach der Dinitrophenyl-Methode bestimmt. Beim Bombyx-Fibroin ergibt sich im festen Faden und nach Lösung in LiJ-Lösung ein durchschnittliches Mol.-Gew. von max. $200000-300000$. In Cupriäthylendiamin-Lösung findet ein Abbau zu einem Mol.-Gew. von etwa 60000 statt. Beim Faden aus Nephila-Fibroin, bei dem keine Entbastung notwendig ist, ergibt sich ebenfalls ein durchschnittliches Mol.-Gew. von max. 200000 - 300 000. In der Spinndrüse hat das Fibroin jedoch ein Mol.-Gew. von nur 30 000. Es wird daher angenommen, daß beim Ausscheiden des Fadens neue Peptidbindungen geknüpft werden. Die Fibroine von Nephila und Bombyx sind hinsichtlich der chemischen Zusammensetzung und des Mol.-Gew. sowie auf dem elektronenmikroskopischen Bild einander sehr ähnlich.
\end{abstract}

$\mathrm{D}$ ie vorliegende Arbeit steht in Zusammenhang mit den funktions-anatomischen Untersuchungen von Peters am Spinn-Apparat der Webespinnen ${ }^{1}$. Sie will einen ersten Beitrag über die biochemischen Grundlagen der Bildung von Spinnfäden geben. Durch Vergleich der Spinnen-Seide mit dem BombyxFibroin möchte sie die Behandlung der Frage von vornherein auf eine breitere Grundlage stellen.

Der Seidenspinner, Bombyx mori, bildet zwei Proteine in derselben Spinndrüse: im hinteren Teil der langen, paarigen Drüsenschläuche wird das Fibroin sezerniert, im mittleren Teil das Sericin. Nach Erreichung des Reifestadiums wird durch Kontraktion der hinteren Partien das Fibroin in das Sericin hineingepreßt, das Tier produziert beim Einspinnen in den Kokon einen Doppelfaden aus Fibroin, der von der Kittsubstanz aus Sericin umhüllt und zusammengehalten wird. Um das saubere Fibroin zu erhalten, muß durch das Verfahren der Entbastung das Sericin entfernt werden.

Bei den Spinnen liegen die Verhältnisse völlig anders. Dort sind eine große Anzahl verschiedener

1 H. M. Peters, Z. Naturforschg. 10 b, 395 [1955].
Spinndrüsen mit getrennten Ausführgängen vorhanden, welche unterschiedliche Fadensorten liefern ${ }^{1}$. Nach seinem Verhalten gegenüber Picrocarminlösung (Merck) zu urteilen, ist das Kokongespinst chemisch nicht einheitlich, die Fäden selbst färben sich bräunlich, die Kittstellen rot (vgl. Abb. 2 und 3 bei Pe ters). Während erstere aus Fibroin bestehen, dürften letztere aus einer sericin-ähnlichen Kittsubstanz bestehen. Wegen ihrer Uneinheitlichkeit sind die Kokonfäden zur Analyse wenig geeignet. In gewissen anderen Spinnfäden hat man aber reines Fibroin vor sich. Es ist dies das von den Glandulae ampullaceae gelieferte Sekret. Diese Fäden braucht die Spinne zur Herstellung ihres Fangnetzes, wobei sie nur für die sogenannten Klebfäden andere Sekrete verwendet. Da die Spinne die oben genannte Fadensorte auch als "Sicherheitsfaden" ausscheidet, wenn sie umherwandert, kann man dieses Material leicht in genügender Menge gewinnen. Es läßt sich nämlich künstlich aus den Spinnwarzen herausziehen (,abspulen“).

Dieses Spinnenfibroin ist für chemische Untersuchungen ein sehr geeignetes Ausgangsmaterial, da hier keine Entbastung notwendig ist. Beim Seiden- 
Gerhard Braunitzer, und Dieter Wolf, Vergleichende chemische Untersuchungen über die Fibroine von Bombyx mori und Nephila madagascariensis (S. 404)

Abb. 1. Fibroin von Bombyx mori in LiJ-Lösung gelöst und dialysiert, bedampft mit Pt-Rh, VergröBerung $1: 60000$.

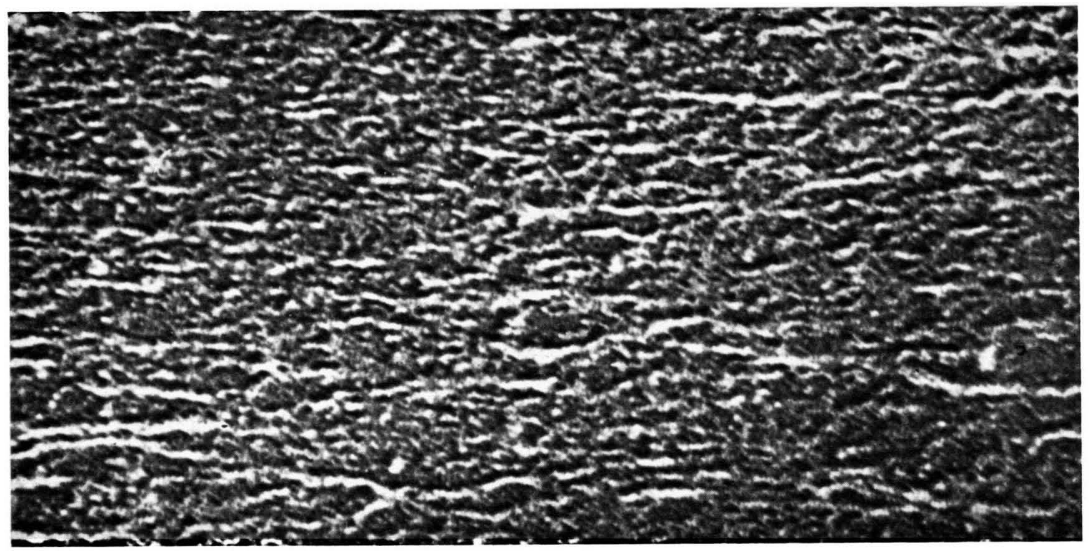

Abb. 2. Fibroin von Nephila madagascariensis, Präparation wie bei Abb. 1. Vergrößerung $1: 60000$.

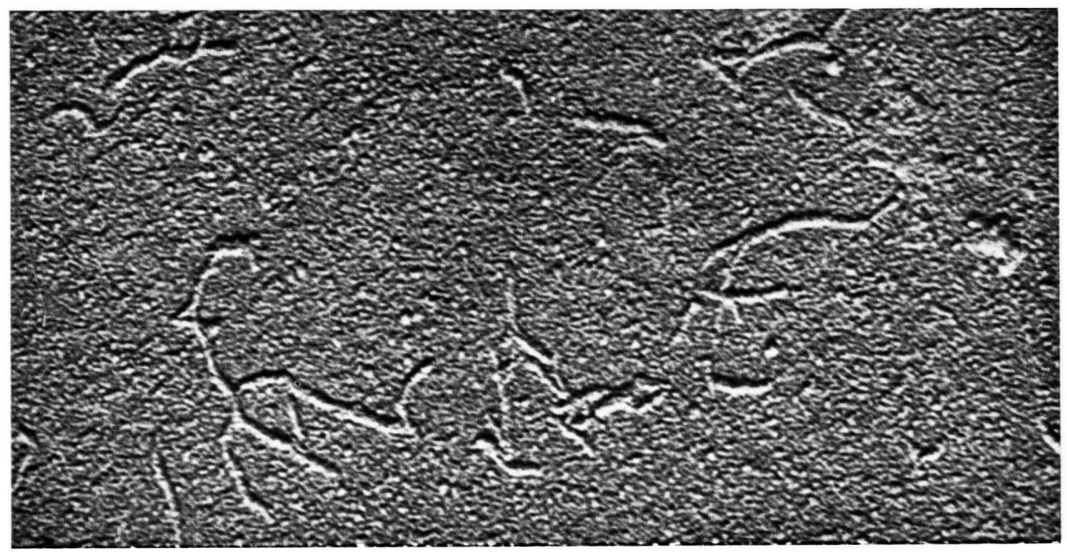

G. Gerber, Vermehrungsweise beim A-Organismus aus der Pleuropneumonie-Gruppe (S. 382)

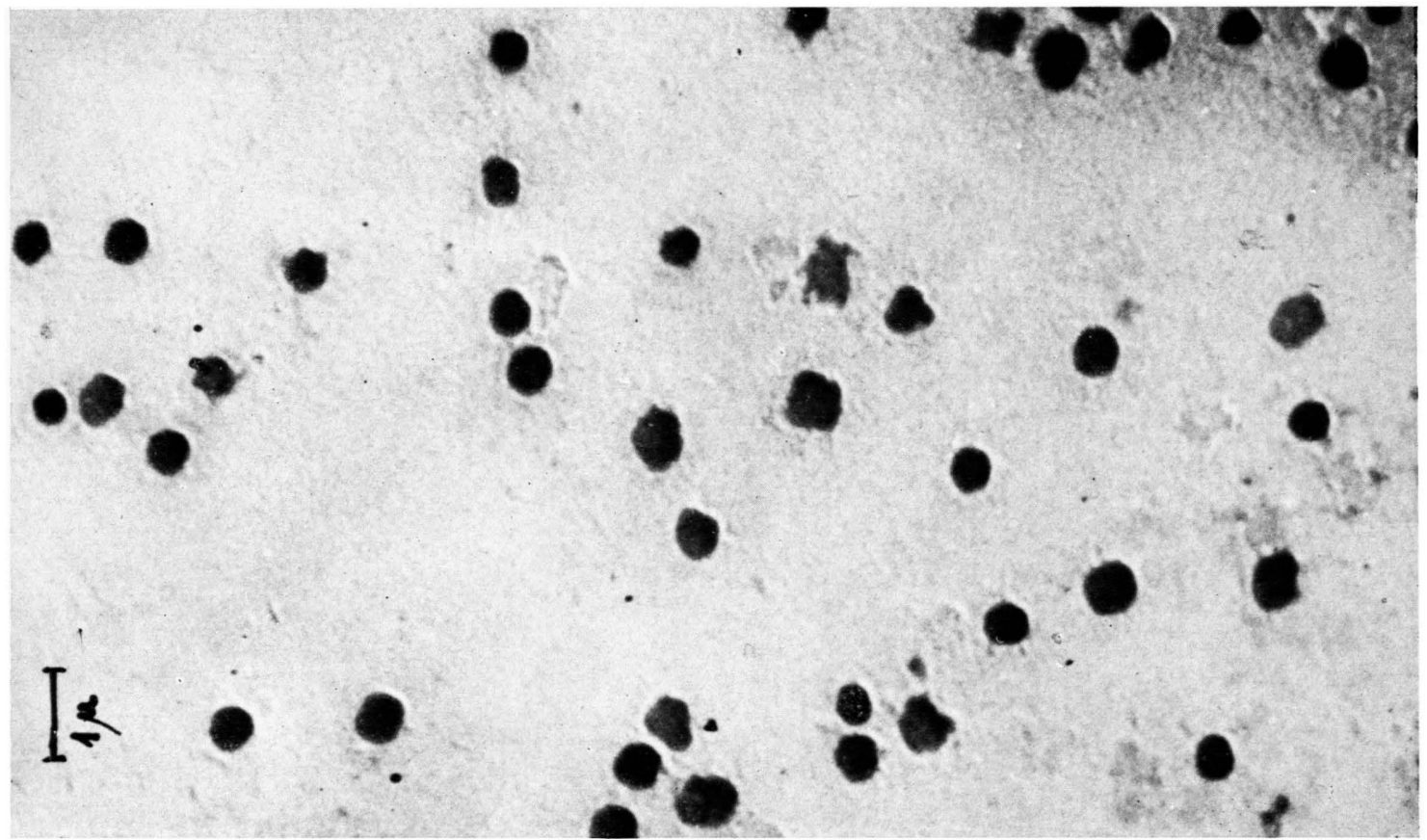

Abb. 2. Elektronenmikroskopische Abbildung von A-Organismen in Zuckerlösung. Fixierung: Osmiumsäure. 


\begin{tabular}{|l|c|c|c|c|c|c|c|c|}
\hline \multirow{3}{*}{ Fibroin } & \multicolumn{7}{|c|}{ Relative Molzahlen bezogen auf Gly = 100 } & \multirow{2}{*}{ Autor } \\
\cline { 2 - 8 } & Gly & Ala & Ser & Val + Leu & Glu + Asp & Threo & Pro & \\
\hline Bombyx mori & 100 & 57,5 & 26,5 & 6,5 & 4,4 & 2,3 & 1,1 & Trista m \\
Bombyx mori & 100 & 62 & 22,5 & 5 & 3,3 & 1,7 & 0,7 & Br. u. W. \\
Nephila & 100 & 80 & 8,75 & 10 & 8,75 & 2 & 2,5 & Br. u. W. \\
\hline
\end{tabular}

Tab. 1. Aminosäuren-Zusammensetzung verschiedener Fibroine.

fibroin bringt dieses Verfahren eine beträchtliche Unsicherheit mit sich, da bei energischem Vorgehen ein Abbau erfolgen kann, bei unvollständiger Entbastung dagegen ein Teil des Sericins beim Fibroin verbleibt ${ }^{2,3,4}$.

Wir benützten für unsere Experimente das Fibroin von Nephila madagascariensis, das bereits im Jahre 1907 von $\mathrm{F}$ is c h e ${ }^{5}$ einer qualitativen Analyse unterworfen wurde. Durch Papierchromatographie sollten zunächst die in beiden Fibroinen vorkommenden Aminosäuren qualitativ und die wichtigsten auch quantitativ bestimmt werden. Weiterhin sollten durch eine vergleichende Bestimmung der Endgruppen die Art der endständigen Aminosäuren und die Mol.Gew. ermittelt werden.

Die qualitative Untersuchung durch zweidimensionale Papierchromatographie ergab, daß die Fibroine hinsichtlich ihrer Zusammensetzung sehr ähnlich sind. Beide enthalten Glykokoll und Alanin als Hauptkomponenten, ferner Tyrosin, Leucin, Isoleucin, Serin, Threonin, Prolin, Phenylalanin, Valin, Lysin, Arginin, Histidin, Glutamin- und Asparaginsäure. Einige Aminosäuren wurden auch quantitativ bestimmt. Hierzu werden sie nach der Hydrolyse in die Dinitrophenyl-(DNP-) Derivate übergeführt, in Anlehnung an das von $\mathrm{Levy}^{6}$ angegebene Verfahren durch zweidimensionale Papierchromatographie getrennt und ihre Extinktion im Beckman-Spektralphotometer gemessen.

Um die Ergebnisse übersichtlich darzustellen, sind in Tab. 1 die Molzahlen der einzelnen Aminosäuren relativ zur Menge des Glykokolls angegeben. Glykokoll wurde willkürlich gleich 100 gesetzt. Man er-

2 R. Signer u. R. Sträss le, Helv. chim. Acta XXX, 155 [1947].

3 R. S i g n er u. R. G l a n z m a n n, Makromol. Chemie 5, 257 [1951].

4 R. G l a n z m a n n u. R. S i g n er, Makromol. Chemie 6, 134 [1952].

5 E. F i s c h e r, Hoppe Seyler's Z. physiol. Chem. 53, 126 [1907]. kennt zunächst, daß die von uns mit der DNPMethode durchgeführte Analyse des Bombyx-Fibroins recht gut mit den in der Literatur ${ }^{7}$ angegebenen Werten übereinstimmt. Das Nephila-Fibroin unterscheidet sich nur wenig vom Bombyx-Fibroin. Es besitzt einen höheren Alanin- und einen niedereren Seringehalt. Weiterhin scheint auch der Gehalt an sauren Aminosäuren etwas höher zu sein als bei Bombyx. Nach Abschluß unserer Versuche wurde uns eine von Lucas, Shaw und $\mathrm{Smith}^{8}$ durchgeführte Analyse des Fibroins von Nephila bekannt, die mit unseren Werten recht gut übereinstimmt. Das Sericin aus Bombyx enthält nach einer orientierenden Analyse sehr viel Serin, daneben aber auch eine große Menge saurer Aminosäuren. Es ist daher unwahrscheinlich, daß der höhere Seringehalt des Bombyx-Fibroins allein auf einer Verunreinigung mit Sericin beruht, da sonst beim Bombyx-Fibroin auch der Gehalt an Glutamin- und Asparaginsäure erhöht sein müßte, was nicht der Fall ist. Das Gemeinsame bei den beiden Fibroinen ist das Vorherrschen der Aminosäuren mit kurzer Seitenkette. Nach Pa uling ist die geringe Raum-Beanspruchung der Seitenkette die Vorbedingung für das Zustandekommen der typischen $\beta$-Struktur der Peptidkette, die wiederum die charakteristischen mechanischen Eigenschaften der Fibroine zur Folge hat.

Die Bestimmung des Mol.-Gew. stößt bei den Fibroinen auf Schwierigkeiten, da bei der Entbastung und Überführung in den löslichen Zustand ein Abbau erfolgen kann. Die günstigen Verhältnisse bei dem Nephila-Fibroin sowie die Verbesserung der Methodik der Endgruppenbestimmung ließen es angezeigt erscheinen, gerade dieser Frage etwas genauer nachzugehen.

6 A. L. L e v y, Nature [London] 174, 126 [1954].

7 C. R. Tris t a m, Advances in Protein Chemistry 5, 83 [1949].

8 F. Lu cas , J. T. B. S h a w u. S. G. S mith, Shirley Institute Memoirs XXVIII, 77 [1955]. 


\begin{tabular}{|c|c|c|c|c|c|c|c|}
\hline Material & Behandlung & Serin & Glu + Asp & Ala & Gly & Threo & Gesamt \\
\hline Bombyx mori-Fibroin & Faden & $\begin{array}{l}0,233 \\
0,211\end{array}$ & $\begin{array}{l}0,059 \\
0,073\end{array}$ & $\begin{array}{l}0,024 \\
0,027\end{array}$ & $\begin{array}{l}0,020 \\
0,035\end{array}$ & $\begin{array}{c}0,017 \\
-\end{array}$ & $\begin{array}{l}0,353 \\
0,346\end{array}$ \\
\hline Bombyx mori-Fibroin & $1 \mathrm{~d}$ in LiJ-Lösung & 0,291 & 0,043 & 0,037 & 0,055 & 0,014 & 0,440 \\
\hline Bombyx mori-Fibroin & $10 \mathrm{~d}$ in LiJ-Lösung & $\begin{array}{l}0,204 \\
0,118\end{array}$ & $\begin{array}{l}0,026 \\
0,042\end{array}$ & - & - & - & $\begin{array}{l}0,230 \\
0,160\end{array}$ \\
\hline Bombyx mori-Fibroin & $\begin{array}{l}\text { in Kupfer (II)-äthylen- } \\
\text { diamin }\end{array}$ & 0,289 & 0,044 & 0,605 & 0,543 & 0,022 & 1,5 \\
\hline Bombyx-Sericin & fest & 0,427 & 0,07 & - & 0,08 & 0,029 & 0,61 \\
\hline Nephila-Fibroin & Faden & 0,06 & 0,088 & 0,098 & 0,013 & - & 0,26 \\
\hline Nephila-Fibroin & Drüseninhalt & $\begin{array}{l}0,199 \\
0,30\end{array}$ & $\begin{array}{l}0,044 \\
0,046\end{array}$ & $\begin{array}{l}2,67 \\
2,97\end{array}$ & $\begin{array}{l}0,023 \\
0,041\end{array}$ & $0, \overline{0} 41$ & $\begin{array}{l}2,9 \\
3,4\end{array}$ \\
\hline
\end{tabular}

Tab. 2. Amino-Endgruppen verschiedener Fibroine in $\mu \mathrm{Mol}$ je $100 \mathrm{mg}$ DNP-Protein.

B e r g m a n n und $\mathrm{N}$ i emann ${ }^{9}$ schlossen aus der Aminosäure-Zusammensetzung, insbesondere dem Gehalt an Histidin auf ein Mol.-Gew. des Seidenfibroins von 217000 . C o l e m a n und $\mathrm{H}$ ow it t 10 führten osmotische Messungen an einer Seide durch, die zunächst in Cupriäthylendiamin gelöst und dann gegen Wasser dialysiert worden war. Sie fanden ein Mol.-Gew. von etwa 30000. $\mathrm{Z}$ a h n ${ }^{11}$ berichtet über Versuche zur Endgruppenbestimmung bei Seidenfibroin. Er fand einen Endgruppengehalt von $0,10-0,14 \%$, was einem Mol.-Gew. von 80000 bis 100000 entsprechen würde. Als Endgruppen wurden Glykokoll und Alanin gefunden. S i g n e $\mathrm{r} 2$ führte Viskositätsmessungen in LiSCN-Lösung durch. Es ergab sich für das Molekül die Form eines Rotationsellipsoids mit den beiden Halbachsen von 460 und $6 \AA$, was einem Mol.-Gew. von 50000 entsprechen würde. Allerdings sind die theoretischen Grundlagen der Berechnung recht unsicher.

Für die Endgruppenbestimmung benützten wir das von $\mathrm{S}$ a n g e r ${ }^{12}$ angegebene Dinitrophenyl-Verfahren, bei dem die endständigen Aminosäuren mit Dinitrofluorbenzol umgesetzt werden und nach anschließender Hydrolyse die Menge an $\alpha$-DNP-Aminosäuren quantitativ auf spektroskopischem Wege gemessen wird.

Wir untersuchten zunächst das Seidenfibroin, und zwar sowohl in Form von entbasteten Fäden als auch nach Lösung in LiJ und Kupfer(II)-äthylendiamin und anschließender Dialyse. Die Ergebnisse sind in Tab. 2 zusammengestellt. Man erkennt zunächst, daß bei der Umsetzung des festen Fadens und des in LiJ gelösten Fibroins annähernd die gleiche Anzahl an Endgruppen erhalten wird. Dies bedeutet, daß bei

9 R. B ergmann u. C. Ni e $m$ a $n n$, J. biol. Chemistry 122, 577 [1938].

10 D. Colem an u. F. O. Howitt, Proc. Roy. Soc. London 190 A, 145 [1947]. der Auflösung in LiJ keine Spaltung von $\alpha$-Peptidbindungen erfolgt. Dieser Befund wurde dadurch unterstrichen, daß bei Seidenfibroin, das 10 Tage in LiJ-Lösung aufbewahrt worden war, die gleiche Endgruppenzahl gefunden wurde wie bei Bestimmung sofort nach dem Auflösen in LiJ. Von den Endgruppen entfallen $60-80 \%$ auf das Serin. Die Endgruppen der einzelnen Moleküle sind also nicht völlig gleichartig. Da der Anteil des Serins an der Gesamtmenge der Aminosäuren relativ gering ist, während er bei den Endgruppen überwiegt, besteht aber doch die Möglichkeit, daß der größere Teil der Fibroinmoleküle wie andere Eiweißstoffe nach einem einheitlichen Plan gebaut ist. Aus der Gesamtzahl der Endgruppen berechnet sich ein durchschnittliches Mol.-Gew. (Zahlenmittel) von max. 200 000-300 000 . Wegen der Unbeständigkeit des DNP-Serins und Glykokolls unter den Bedingungen der Hydrolyse stellt dieser Wert ein Maximum dar. Er könnte auch bis zu $20 \%$ niedriger sein.

Bei geraden unverzweigten Peptidketten muß die Zahl der Amino-Endgruppen mit der der CarboxylEndgruppen übereinstimmen. Es wurde daher auch ein vorläufiger Versuch zur Bestimmung der Carboxyl-Endgruppen durch Hydrazinspaltung nach A k a b o r i ${ }^{13}$ unternommen. Wir fanden als C-terminale Gruppen Serin, Alanin, Glykokoll, insgesamt $0,3-0,4 \mu \mathrm{Mol}$ je $100 \mathrm{mg}$ Protein. Die Übereinstimmung mit der Zahl der Amino-Endgruppen ist also

11 H. Z a h n, Textil Rundschau 9, 119 [1954] u. priv. Mitt.

12 F. S a n g e r, Biochem. J. 39, 507 [1945].

13 S. Akabori, K. Ohno u. K. Narita, Bull. Chem. Soc. Japan 25, 214 [1952]. 
befriedigend. Allerdings ist bei dieser Analyse der Korrekturfaktor für die Berechnung gerade bei den gegebenen Aminosäuren ziemlich hoch, so daß die Analyse nicht sehr sicher ist.

Nach Lösung des Fibroins in Kupfer(II)-äthylendiamin und Dialyse nach dem Verfahren von Coleman und Howitt ${ }^{10}$ fanden wir ein DurchschnittsMol.-Gew. von 60000 , die Größenordnung ist also dieselbe wie bei diesen Autoren. Die Zahl der SerinEndgruppen bleibt in diesem Falle konstant, während zusätzlich Alanin- und Glykokoll-Endgruppen auftreten. Es ergibt sich also eindeutig, daß in diesem Lösungsmittel ein Abbau stattfindet, wie es auch schon von $\mathrm{S}$ ig $\mathrm{n}$ e $\mathrm{r}^{2}$ festgestellt wurde.

Parallel zu dem Bombyx-Fibroin wurde auch das Sericin untersucht, für das sich ein mittleres Mol.Gew. von 160000 ergab.

Bei dem Nephila-Fibroin wurde aus Mangel an Material nur der feste Faden untersucht. Die Art der Endgruppen unterscheidet sich vom BombyxFibroin, da bei Nephila Serin, Glutaminsäure + Asparaginsäure und Alanin in etwa gleichen Mengen auftreten. Wir fanden jedoch etwa das gleiche mittlere Mol.-Gew. von etwa 300 000. Da das NephilaFibroin weder entbastet noch einem Lösungsmittel ausgesetzt wurde, ist dieser Wert als besonders zuverlässig anzusehen.

Mit dem hohen Mol.-Gew. steht in Übereinstimmung, daß man in den gelösten Fibroinen von $\mathrm{Ne}$ phila und Bombyx elektronenmikroskopisch gut ausgebildete Fäden erkennt, die einen Durchmesser von etwa $100 \AA$ besitzen. Es handelt sich vermutlich um Aggregate von Peptidketten, eine Beziehung zu den Dimensionen der Grundmoleküle konnte bisher noch nicht festgelegt werden (Abb. $1^{*}$ und 2).

Neben dem abgespulten fertigen Fibroin wurde auch der Inhalt der betreffenden Drüsen (Glandulae ampullaceae) untersucht ${ }^{14}$. Überraschenderweise ergab sich bei diesem ein etwa 10-mal höherer Gehalt an Endgruppen, die zu 90\% aus Alanin bestehen, und damit ein durchschnittliches Mol.-Gew. von nur 30 000. Da der Fibroinfaden unmittelbar aus dem Drüseninhalt stammt, müßten sich Verunreinigungen bei beiden Bestimmungen in gleicher Weise auswirken. Zur Erklärung des überraschenden Befunds ist man zur Annahme gezwungen, daß beim Ausschei-

14 Zwischen dem Gl.amp. majores und minores wurde kein Unterschied gemacht.

* Abb. 1 u. 2. s. Tafel S. 404 a.

** Anmerkung P e ter s : Nach dem anatomischen Bau der Glandulae ampullaceae mit ihren Ausführgängen könnte man sich sehr gut vorstellen, daß die Elementar- den des Fadens neue Peptidbindungen hauptsächlich am Alaninrest geknüpft werden. Theoretisch wäre eine derartige Peptidsynthese möglich, da ja die Proteinkonzentration sehr hoch ist und auch die Mitwirkung von Proteinasen oder Peptidasen an dem Vorgang nicht ausgeschlossen ist. Es wäre daher wünschenswert, wenn der Spinnvorgang und die dabei erfolgenden Veränderungen an den Fibroinmolekülen näher untersucht würden ${ }^{* *}$.

Zusammenfassend läßt sich sagen, daß die Fibroine von Bombyx und von Nephila einander auffallend ähnlich sind. Die Ähnlichkeit erstreckt sich auf die wesentlichen Kennzeichen der chemischen Zusammensetzung, auf das Mol.-Gew. und auf das elektronenmikroskopische Bild. $\mathrm{Zu}$ beachten bleibt dabei, daß es sich bei den untersuchten Fäden des Seidenspinners um Kokon-Gespinst handelt, bei denen der Spinne jedoch um Fäden, die zur Herstellung des Netzes oder als "Sicherheitsfäden“ benützt werden.

\section{Experimenteller Teil}

Fibroin von Nephila

Über die Herkunft unseres Spinnen-Materials hat bereits Peters 1 berichtet. Durch „Abspulen“ der Tiere konnten wir in einem Fall bis $70 \mathrm{mg}$ Spinnfäden gewinnen. Der abgespulte Faden besteht mikroskopisch aus mehreren, bis vier, Einzelfäden, zwei etwa $6 \mu$ stark, zwei von etwas geringerem Durchmesser.

Um den Drüseninhalt selbst zu gewinnen, wurden die Glandulae ampullaceae durch Präparation freigelegt. Dann wurde der bauchige Teil der Drüsen (der Sammelbehälter) herausgenommen und sofort in 10-proz. Kaliumcarbonat-Lösung gegeben. Das Sekret stellt eine glasklare, zähe Masse dar. Sie ist von der sehr dünnen Wandung der Drüsenampulle umgeben. Auch wenn diese platzt, behält die Sekretmasse ihre Form und kann mit der Pinzette erfaßt und in das Gefäß mit der Lösungsflüssigkeit übergeführt werden. Wenn das Wandepithel nicht schon bei der Präparation abgegangen war, wurde es belassen, da es im Verhältnis zum Sekret mengenmäßig überhaupt nicht ins Gewicht fällt. Nach 3-4 Stdn. war das Sekret zu einer orangegelben Flüssigkeit gelöst. Schütteln auf der Schüttelmaschine ist zu vermeiden, der Drüseninhalt darf auch nicht an der Luft getrocknet oder erstarrt sein, da er sich sonst nicht mehr mit Carbonat in Lösung bringen läßt.

\section{Fibroin von $B$ o $m b y x$}

Zur Entbastung wurden die gelben Kokons von Bombyx mori 2-3-mal in 4-proz. Sodalösung kurzzeitig auf

teilchen aus dem Sammelbehälter der Drüse auf dem Weg zur Düse zu höheren Einheiten zusammentreten, vielleicht unter der Wirkung von Fermenten, die der Wandung des Ausführganges entstammen ***. Diese Annahme würde die auffallende Länge des ausleitenden Kanals verständlich machen (vgl. P e te r s S. 398 u. Abb. 5).

*** Vgl. Pe t e r s Fußnote 16. 
$70^{\circ}$ erwärmt. Das Sericin und ein gelber Farbstoff ließen sich auf diese Weise leicht entfernen. Nach Auswaschen mit Wasser, Äthylalkohol und Äther sowie 24-stdg. Trocknen erhält man ein weißes Fibroin, das nur noch wenig Sericin enthält, wie die mikroskopische Kontrolle mit Picrocarmin zeigte.

\section{Sericin von $B$ o $m b y x$}

Zerschnittene Kokons wurden in Wasser eine Stde. auf $50^{\circ}$ erhitzt, das Sericin wird dann abfiltriert und lyophylisiert. Man erhält einen voluminösen, leicht gelb gefärbten Stoff, der bei Zimmertemperatur in Wasser nur schlecht löslich ist.

\section{Auflösung der Fibroine}

Um eine wäßrige Lösung von Fibroin zu erhalten, benützten wir konzentrierte Lösungen von LiJ oder Kupfer(II)-äthylendiamin, die anschließend dialysiert wurden. $100 \mathrm{~g}$ LiJ wurden in $100 \mathrm{ccm}$ Wasser gelöst, schwach erhitzt und das Fibroin hineingegeben, das sich sofort zu einer 1-2-proz. Lösung auflöst. Die Dialyse dauerte 10 bis 20 Stunden. Die Kupfer(II)-äthylendiamin-Lösungen wurden nach der Vorschrift von $\mathrm{Colem}$ a n und $\mathrm{Ho}$ w it t 10 hergestellt. Das Fibroin löst sich hierin schnell. Die entstandene Lösung wurde dann ohne Neutralisation dialysiert. Ein Teil des Fibroins fiel hierbei aus, er wurde verworfen.

Bestimmung der Aminosäure-

$\mathrm{Zus}$ a mimensetzung

Zur qualitativen Analyse wurde das Protein im allgemeinen $16 \mathrm{Stdn}$. bei $110^{\circ} \mathrm{C}$ mit $6-n$. $\mathrm{HCl}$ im verschlossenen Rohr hydrolysiert. Anschließend wurde in der üblichen Weise zweidimensional chromatographiert. Wir verwendeten in der einen Dimension Butanol/Essigsäure/ Wasser $4: 1: 5$, in der anderen Phenol/Wasser $8: 2$.

Zur quantitativen Bestimmung wurde das wie oben hergestellte Hydrolysat nach dem Abdampfen der Salzsäure mit Fluordinitrobenzol nach $\mathrm{S}$ a n ger 12 umgesetzt, und zwar in wäßrig-alkoholischer Lösung. Die Reaktionsdauer betrug 2 Stdn., anschließend wurde die alkalische Lösung ausgeäthert, dann angesäuert und die DNP-Aminosäuren durch 3-maliges Schütteln mit Essigester extrahiert. Zur weiteren Reinigung wurden die DNP-Aminosäuren nochmals in Bicarbonatlösung übergeführt, die nach dem Ansäuern wiederum mit Essigester extrahiert wurde. Nach dem Abdampfen des Essigesters wurde das bei dem Umsatz gebildete Dinitrophenol nach Mills ${ }^{15}$ im Vakuum abdestilliert. Dann wurden nach einem Verfahren ähnlich dem von Levy ${ }^{6}$ die DNPAminosäuren durch zweidimensionale Papierchromatogra-

15 G. L. M ills, Biochem. J. 50, 707 [1952]. phie getrennt. In der einen Richtung wurde Butanol/ 0,1-proz. $\mathrm{NH}_{3}$, in der anderen 1,5-m. Phosphatpuffer benützt.

\section{Bestimmung der Amino-Endgruppen}

Für die Umsetzung mit Dinitrofluorbenzol wurde das feste Fadenmaterial in gesättigter Bicarbonatlösung suspendiert (etwa $50 \mathrm{mg}$ Fibroin in $1 \mathrm{ccm}$ ). Bei den gelösten Fibroinen wurde nach der Dialyse festes Bicarbonat bis zur Sättigung hinzugefügt. Dann wurden zwei Volumina Äthanol, das 5\% Dinitrofluorbenzol enthielt, zugegeben. Hierbei fällt ein Teil des Bicarbonats aus. Die Mischung wurde mit Glaskugeln versetzt und auf einer Schüttelmaschine 2 Stdn. kräftig geschüttelt. Am Ende der Reaktion war das DNP-Fibroin vollständig ausgefallen, es wurde abfiltriert und mit Wasser, Äthanol und Äther gewaschen, bis das Filtrat farblos war. Auf diese Weise wird das während der Reaktion entstehende Dinitrophenol vollständig entfernt. Das DNP-Fibroin wurde dann bei $70^{\circ} \mathrm{C}$ im Vakuum getrocknet.

Zur Hydrolyse wurde ein Teil mit 100 Teilen $6-m . \mathrm{HCl}$ im Einschlußrohr 8-16 Stdn. auf $110^{\circ}$ erhitzt. Das Hydrolysat wurde mit dem 4-6-fachen Volumen an Wasser verdünnt und die DNP-Aminosäuren durch 3-malige Extraktion mit Äther extrahiert. Unter diesen Umständen könnten in der wäßrigen Phase nur DNP-Histidin und DNP-Arginin zurückbleiben, sie waren jedoch beide nicht nachweisbar. Der Ätherextrakt wurde 3-mal mit 1- $n$. HCl gewaschen, die Salzsäureanteile wurden vereinigt und 1-mal mit Äther ausgeschüttelt. Die Ätherextrakte wurden eingedampft und die DNP-Aminosäuren nach dem oben beschriebenen Verfahren in Anlehnung an Lev y ${ }^{6}$ quantitativ bestimmt.

Die Bestimmung der Carboxyl-

End gruppen

$100 \mathrm{mg}$ Fibroin wurden in $1 \mathrm{ccm}$ Hydrazin gelöst und $8 \mathrm{Stdn}$. auf $100^{\circ} \mathrm{C}$ erhitzt. Die Lösung des Proteins vollzog sich nur schwer und war auch nach der Beendigung der Reaktionszeit nicht vollständig. Das Reaktionsgemisch wurde mit einigen Tropfen Wasser in eine flache Schale gespült, im Exsikkator über $\mathrm{H}_{2} \mathrm{SO}_{4}$ bei $1 \mathrm{~mm} \mathrm{Hg}$ Druck das überschüssige Hydrazin entfernt, der Rückstand in $5 \mathrm{ccm} \mathrm{m} / 15$-Phosphat aufgelöst und mit $2 \mathrm{ccm}$ Benzaldehyd $1 / 2$ Stde. geschüttelt. Das Reaktionsgemisch wurde 3-mal ausgeäthert, filtriert und das Filtrat wie oben mit Dinitrofluorbenzol umgesetzt.

Wir danken der Deutschen Forschungsgemeinschaft für die Unterstützung der Arbeiten, insbesondere für das D. W. erteilte Stipendium.

Herrn Prof. Dr. G. S c h r a m m danken wir für die Förderung der Arbeit, Herrn Prof. Dr. H. Z a h n, Chemisches Institut, Heidelberg, für wertvolle Hinweise. 